\title{
Research on Dynamic Vehicle Model Equipped Active Stabilizer Bar
}

\author{
Anh Nguyen Tuan*, ${ }^{*}$, Binh Hoang Thang ${ }^{2}$ \\ ${ }^{1}$ Mechatronics-Automobile Department, Ha Noi University of Business and Technology, Viet Nam \\ ${ }^{2}$ Automotive Engineering Department, Ha Noi University of Science and Technology, Viet Nam
}

\begin{tabular}{l} 
A R T I C L E I N F O \\
\hline Article history: \\
Received: 30 May, 2019 \\
Accepted: 27 July, 2019 \\
Online: 07 August, 2019 \\
\hline Keywords: \\
Active stabilizer bar \\
Rollover \\
Anti-roll moment \\
Roll angle \\
Dynamic vehicle model \\
\hline
\end{tabular}

\section{Introduction}

\subsection{Rollover of the vehicle}

When the vehicle goes on the road with high speed and the driver suddenly changes the direction, the centrifugal force appears. This force tends to go out of the rotating arc, cause the center of sprung mass to change, the moment causes the rolled vehicle will appear, denoted by $\mathrm{M}_{1}[1]$.

$$
M_{1}=M_{F}+M_{P}
$$

Where:

$$
\begin{aligned}
& M_{F} \text { : Moment of centrifugal force. } \\
& M_{P} \text { : Moment of gravitation. }
\end{aligned}
$$

Assume that $\mathrm{M}_{2}$ is the anti-roll moment of the vehicle, this moment is caused by the suspension system.

If:

$\mathrm{M}_{2}<\mathrm{M}_{1} \leq \mathrm{M}_{2 \max }$ : The vehicle body is tilted.

$\mathrm{M}_{1}>\mathrm{M}_{2 \max }$ : The vehicle is rollover.

Therefore, to limit the rollover of the vehicle is necessary to reduce the moment $\mathrm{M}_{1}$. In fact, this value can't control, the alternative solution is to increase the value of anti-roll moment $\mathrm{M}_{2}$ by adding an extra elastic part [2].
The problem of horizontal instability of the vehicle has been studied by foreign scientists as well as proposed many options, one of the solutions offered is to use stabilizer bar [3, 4]. However, previous studies often concentrate on the passive stabilizer bar on the vehicle with a linear single-track model and a half model [5]. This paper focuses on establishing space dynamic vehicle model equipped active stabilizer bar to limit the roll angle of the vehicle when steering.

\subsection{Equipped stabilizer bar on the vehicle}

To increase the value of the anti-roll moment $\mathrm{M}_{2}$ is necessary to increase the stiffness of the suspension system. Today, vehicles are often equipped the stabilizer bar to link the two wheels of an axle [6]. The stiffness and size of the stabilizer bar are proportional to the vehicle's mass, the larger vehicle requires a stabilizer bar with a larger diameter and harder. Therefore, it will have a significant impact on the smoothness of the vehicle when moving on the road.

With the above problem, instead of using normal mechanical stabilizer bar, some middle-class and high-class vehicles were equipped stabilizer bar with electric or hydraulic control. The active stabilizer bar has the advantage of reducing the roll angle when steering and limiting the impact to the smoothness when moving on the bumpy road.

*Anh Nguyen Tuan, Email: taae177@gmail.com 


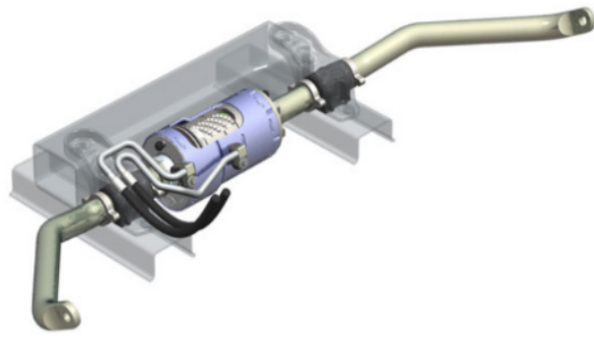

Figure 1: Active stabilizer bar

\section{Dynamic vehicle model}

\subsection{Dynamic vehicle model 7 DOF}

Consider the model of the vehicle to show in Figure 2. This section, the oscillation includes the vertical oscillation $(\mathrm{z})$ and the horizontal oscillation $\left(\varphi_{\mathrm{x}}\right)$ because the vehicle steers at a stable velocity and the road surface are considered to be flat, it is possible to ignore longitudinal oscillation of the vehicle $\left(\varphi_{\mathrm{y}}\right)$.

Suppose that:

$$
\begin{aligned}
& F_{1}=F_{C 11}+F_{K 11}+F_{C 21}+F_{K 21} \\
& F_{2}=F_{C 12}+F_{K 12}+F_{C 22}+F_{K 22}
\end{aligned}
$$

Using the method of separation of objects, according to [7] equations describing vertical displacement and roll angle of suspended mass:

$$
\begin{gathered}
m \ddot{z}=F_{1}+F_{2} \\
\left(I_{x}+m h_{1}^{2}\right) \ddot{\varphi}_{x}=\left(F_{1}-F_{2}\right) b+M_{1}-M_{S B}
\end{gathered}
$$

Where:

$\mathrm{M}_{1}$ : Moment of roll vehicle.

$$
M_{1}=\left(a_{y} \cos ^{2} \varphi_{x}+g \sin \varphi_{x}\right) m h_{1}
$$

$\mathrm{M}_{\mathrm{SB}}$ : Moment of the stabilizer bar.

$$
M_{S B}=m g h_{l} \sin \varphi_{x}
$$

$\mathrm{I}_{\mathrm{x}}$ : Moment of inertia around the $\mathrm{x}$-axis.

$h_{1}$ : The distance from the center of gravity to the roll axis. mass:

The equation describes the vertical displacement of unsprung

$$
m_{i j} \ddot{\xi}_{i j}=F_{K L i j}-F_{C L i j}-F_{K i j}
$$

According to $[8,9]$

The elastic force of spring:

$$
F_{K i j}=K_{i j}\left(\xi_{i j}-z \pm b \varphi_{x}\right)
$$

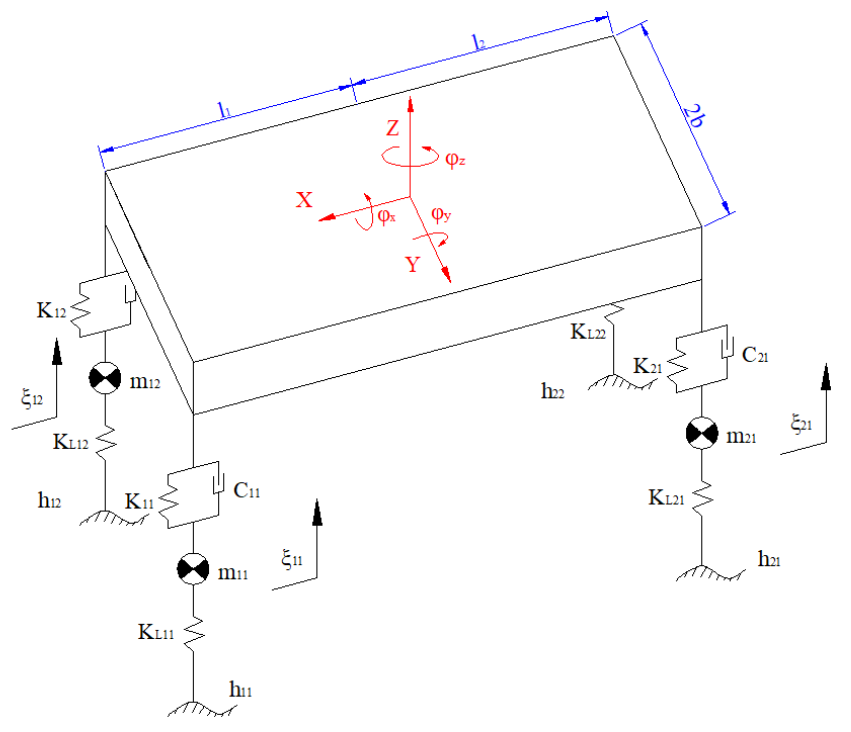

Figure 2: Dynamic vehicle model 7 DOF

The resistive force of damping:

$$
F_{C i j}=C_{i j}\left(\dot{\xi}_{i j}-\dot{z} \pm b \dot{\varphi}_{x}\right)
$$

The elastic force of tire:

$$
F_{K L i j}=K_{L i j}\left(h_{i j}-\xi_{i j}\right)
$$

\subsection{Double track dynamic vehicle model}

The body vehicle is tilted caused by lateral acceleration when steering. Place the dynamic vehicle model 7 DOF on the road plane (Oxy), the vehicle performs 3 movements include longitudinal movement (x), lateral movement (y) and movement around the vertical axis $\left(\varphi_{z}\right)$. For each of the above unknowns, there will be a corresponding equation describing the movement of the vehicle.

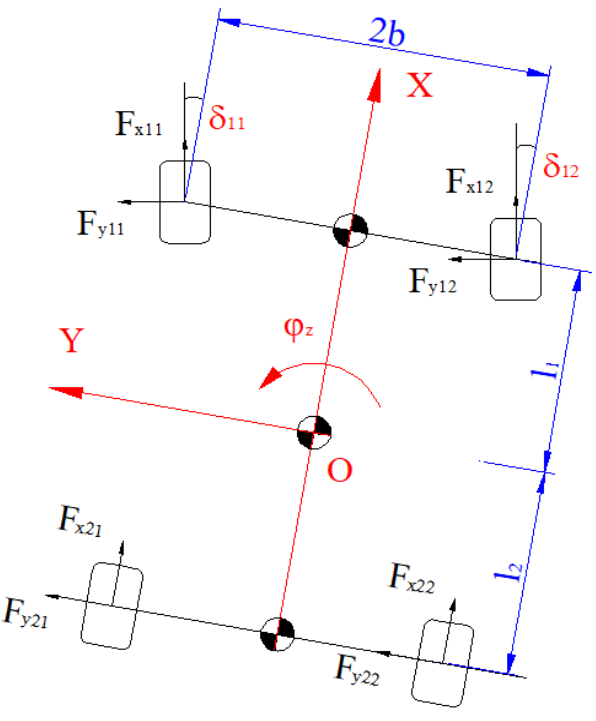

Figure 3: Double track dynamic vehicle model 
Assume that the steering wheel angle $\delta$ is very small and ignore $\mathrm{M}_{\mathrm{z}}$ at the wheel. According to [10] the equation describes the movement of the vehicle in the case of constant velocity in the form:

$$
\begin{gathered}
M\left(\dot{v}_{y}+\dot{\varphi}_{z} v_{x}\right)=F_{y 1}+F_{y 2} \\
I_{z} \ddot{\varphi}_{z}=F_{y l} l_{1}-F_{y 2} l_{2}
\end{gathered}
$$

With:

$$
\begin{aligned}
& F_{y 1}=F_{y 11}+F_{y 12} \\
& F_{y 2}=F_{y 21}+F_{y 22}
\end{aligned}
$$

If the deformation wheel is considered in the linear domain, the lateral force of the wheel is determined by the following formula:

$$
F_{y}=-C_{\alpha} \alpha
$$

Where:

$\mathrm{C}_{\alpha}$ : Side stiffness of the tire.

$\alpha$ : Slip angle of the tire.

Table 1: Specifications of the vehicle

\begin{tabular}{|c|c|c|}
\hline Description & Symbol & Value \\
\hline Distance from center to front axle & $1_{1}$ & $\begin{array}{c}1110 \\
(\mathrm{~mm})\end{array}$ \\
\hline Distance from center to rear axle & $1_{2}$ & $\begin{array}{c}1655 \\
(\mathrm{~mm})\end{array}$ \\
\hline Base width & $2 \mathrm{~b}$ & $\begin{array}{c}1500 \\
(\mathrm{~mm})\end{array}$ \\
\hline $\begin{array}{c}\text { Distance from center of gravity to } \\
\text { roll center }\end{array}$ & $\mathrm{h}_{1}$ & $\begin{array}{c}480 \\
(\mathrm{~mm})\end{array}$ \\
\hline Sprung mass & $\mathrm{m}$ & $\begin{array}{c}1700 \\
(\mathrm{~kg})\end{array}$ \\
\hline Unsprung mass & $\mathrm{m}_{\mathrm{ij}}$ & $\begin{array}{c}45 \\
(\mathrm{~kg})\end{array}$ \\
\hline
\end{tabular}

\section{Simulate the oscillation of the vehicle}

\subsection{The dependence of the roll angle on the velocity}

Steering angle I as shown in Figure 4, the simulation is carried out when the vehicle is moving with velocities $\mathrm{v}_{1}=60 \mathrm{~km} / \mathrm{h}$ and $\mathrm{v}_{2}=90 \mathrm{~km} / \mathrm{h}$.

From the graph in Figure 5, it can be seen that at $\mathrm{v}=60 \mathrm{~km} / \mathrm{h}$, when the equipped with passive stabilizer bar, the roll angle decreases from $3.8^{0}$ to $3.1^{0}$. If the velocity increases to $90 \mathrm{~km} / \mathrm{h}$, this value, in turn, decreases from $5.2^{0}$ to $4.3^{0}$. Therefore, it can be concluded that the vehicle's roll angle depends on velocity.

\subsection{The dependence of the roll angle on the central height}

For vehicles with a high center of gravity, the distance from the center of gravity to roll center is changed, this affects the vehicle's roll angle when steering. In the condition of the vehicle moving at the velocity $\mathrm{v}=60 \mathrm{~km} / \mathrm{h}$, the steering angle is as shown in Figure 4, the correlation between the roll angle $\varphi_{\mathrm{x}}$ and the distance $h_{1}$ is described through the graph below.

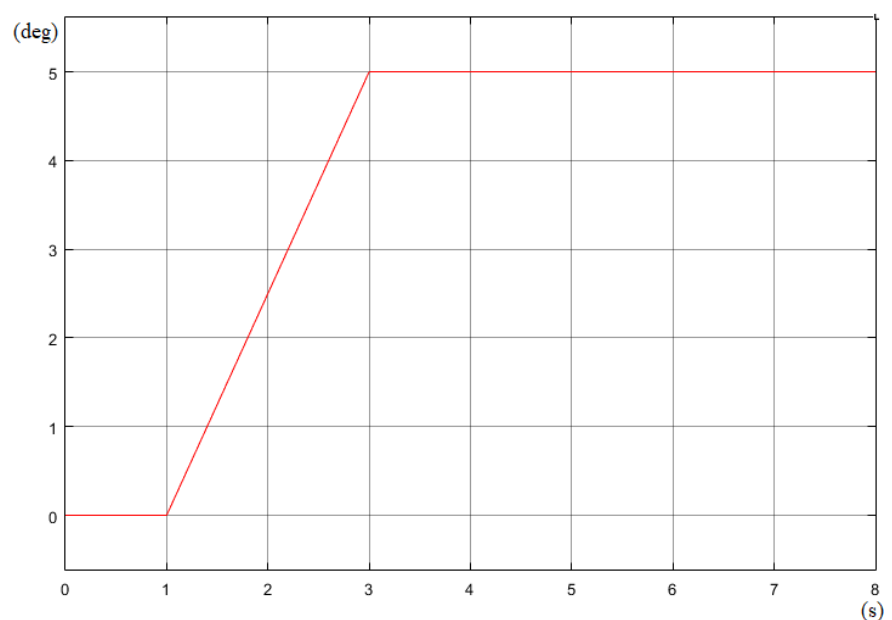

Figure 4: Steering angle I

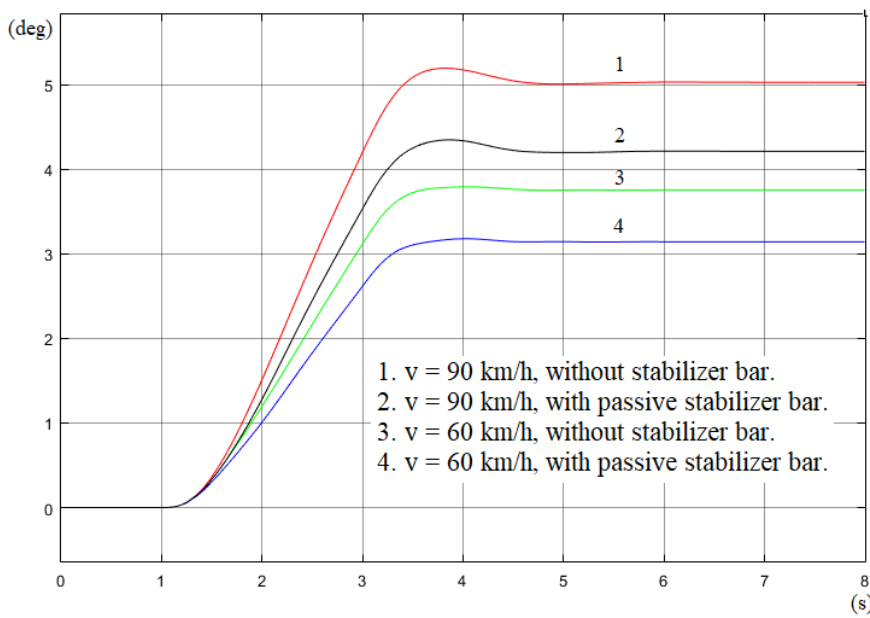

Figure 5: Roll angle depends on velocity

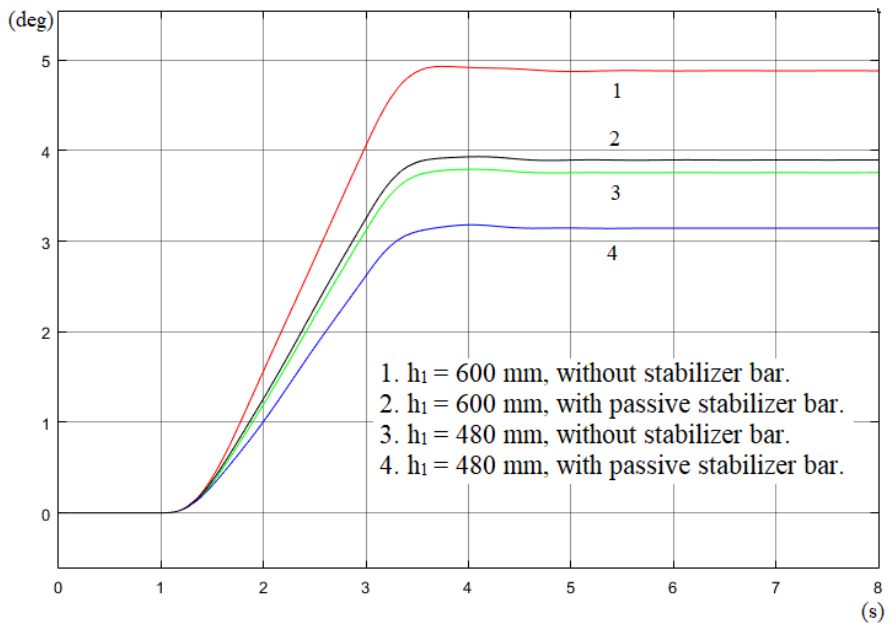

Figure 6: Roll angle depends on $\mathrm{h}_{1}$

In the graph in Figure 6, when the vehicle is equipped the stabilizer bar is the vehicle's roll angle $\varphi_{\mathrm{x}}$ decreases from $3.8^{0}$ to $3.1^{0}$ with the distance $\mathrm{h}_{1}=0.48 \mathrm{~m}$. When the height of vehicle increases, the distance between the center of gravity and roll center $\mathrm{h}_{1}=0.6 \mathrm{~m}$, the corresponding angle value is $4.9^{0}$ and $3.9^{0}$. 
Therefore, large-sized vehicles are often unstable when steering at high velocity.

\subsection{The dependence of the roll angle on the steering angle}

In the condition of driving with a larger angle $\delta$ as shown in Figure 7 at $\mathrm{v}=60 \mathrm{~km} / \mathrm{h}$, the dependence between the vehicle's roll angle and steering angle is shown in Figure 8.

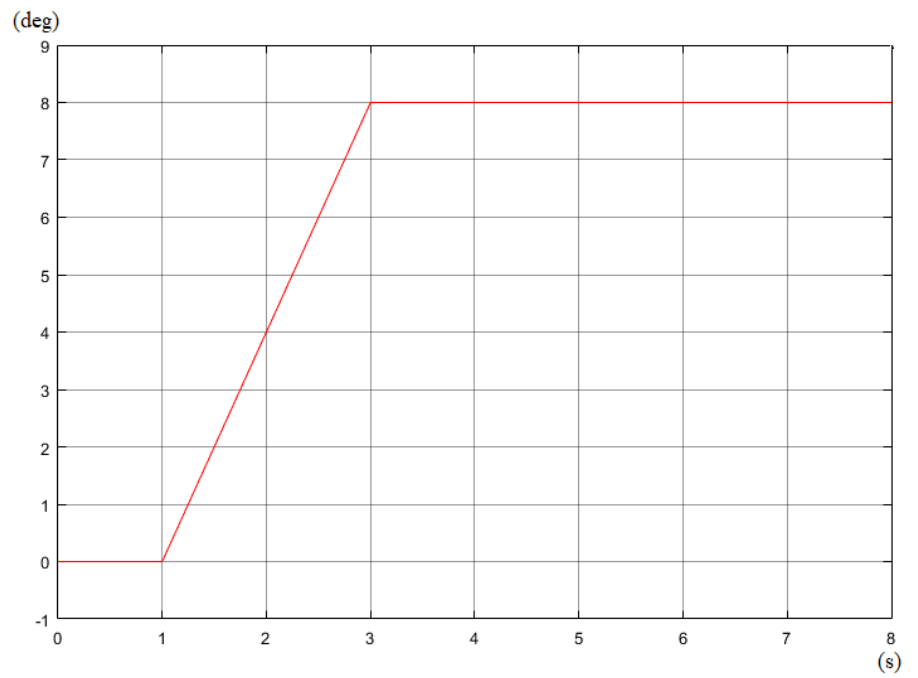

Figure 7: Steering angle II

When the value of steering angle $\delta$ increases, it also means that the roll angle of the vehicle $\varphi_{\mathrm{x}}$ increases. If the vehicle has the stabilizer bar, it will greatly reduce this value.

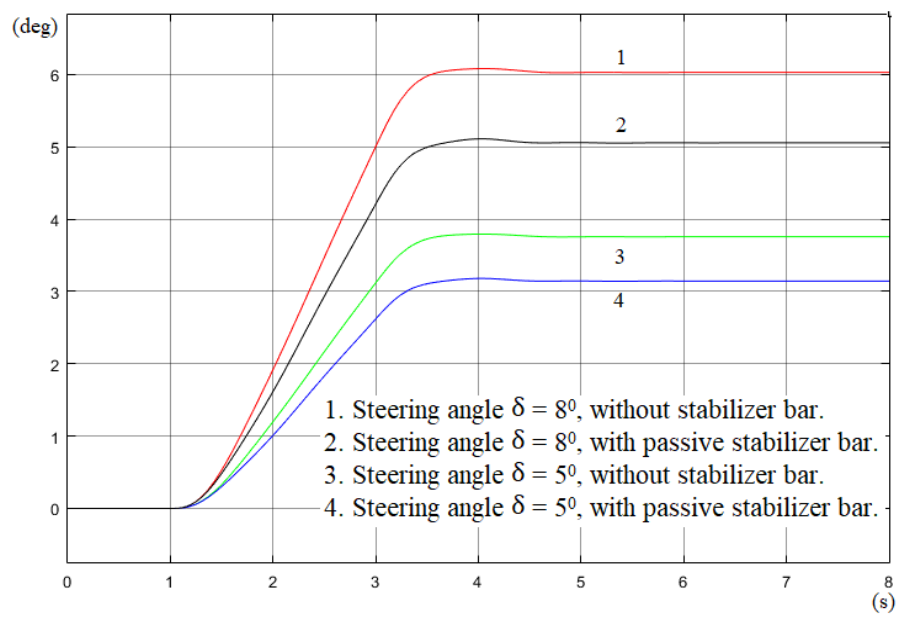

Figure 8: Roll angle depends on steering angle

From the graph in Figure 5, Figure 6 and Figure 8, it can be seen that when the vehicle has a stabilizer bar, the vehicle's roll angle will decrease depending on the working mode. If equipped with a passive stabilizer bar, there will be some disadvantages as follows:

+ The small size bar will not be able to withstand the constant load change, especially for high-mass, high-velocity vehicles.

+ The big size bar will be difficult to arrange in the vehicle and increase the mass isn't suspended. Therefore, it causes strong fluctuations when entering the bumpy road.
+ The ability to minimize the vehicle's roll angle is limited in dangerous situations.

For these reasons, today active stabilizer bar is gradually put to use in the middle-class and high-class vehicle.

\subsection{The vehicle equipped active stabilizer bar}

The active stabilizer bar is controlled based on input parameters such as vehicle's roll angle, center height, displacement of the suspension system,... Control rules are established based on basic mathematical models such as linear models, nonlinear models, static models, dynamic models,... In this study, the moment by the active stabilizer bar is controlled according to the following rule:

At the first mode

$$
\begin{aligned}
& M_{A}=C_{t} C_{k I} \sin \left(\varphi_{x}-2\right) \sin \varphi_{x} \\
& \varphi_{x}-2=\left\{\begin{array}{l}
\varphi_{x}-2, \varphi_{x} \geq 2 \\
0, \varphi_{x}<2
\end{array}\right.
\end{aligned}
$$

At the second mode

$$
\begin{aligned}
& M_{A}=C_{t}\left[1.3+C_{k 2} \sin \left(\varphi_{x}-4\right)\right] \sin \varphi_{x} \\
& \varphi_{x}-4=\left\{\begin{array}{l}
2, \varphi_{x} \geq 6 \\
\varphi_{x}-4, \varphi_{x}<6
\end{array}\right.
\end{aligned}
$$

where:

$\mathrm{M}_{\mathrm{A}}$ : Moment of the active stabilizer bar.

$\mathrm{C}_{\mathrm{t}}$ : Control coefficient; $\mathrm{C}_{\mathrm{t}}=11000$.

$\mathrm{C}_{\mathrm{k}}$ : Activation coefficient.

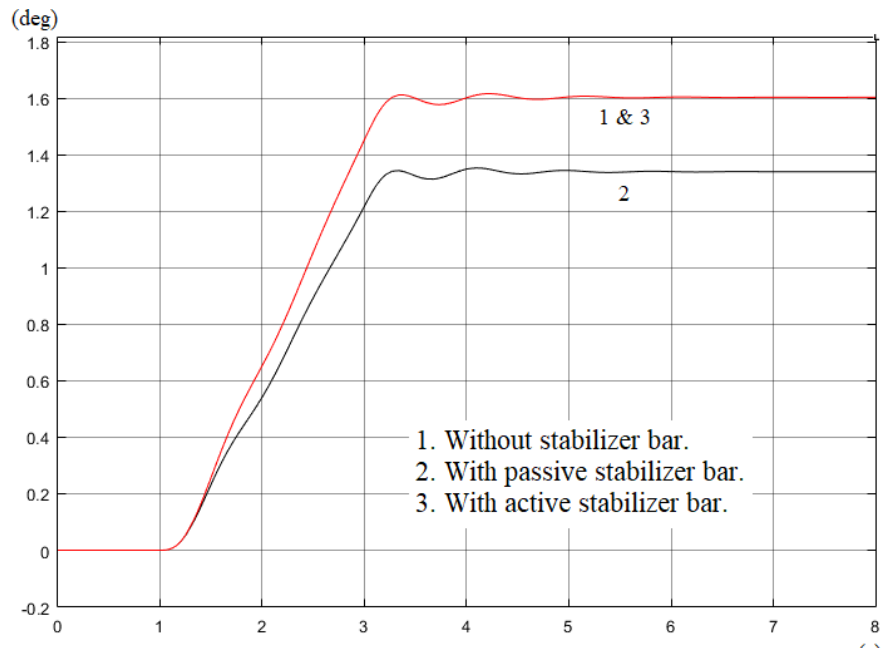

Figure 9: Roll angle at $\mathrm{v}=30 \mathrm{~km} / \mathrm{h}$

When the vehicle's roll angle $\varphi_{x}<2.0^{\circ}$, the stabilizer bar will not activate. If the roll angle exceeds this value, it will activate at (1) corresponding to the coefficient $\mathrm{C}_{\mathrm{k} 1}=27$. In case of vehicle's roll angle value $\varphi_{\mathrm{x}}>4.0^{0}$, the active stabilizer bar will activate at (2) corresponding to the coefficient $\mathrm{C}_{\mathrm{k} 2}=45$, the value of the anti- 
roll moment that the bar providers is increased more so that the vehicle's roll angle is significantly reduced.

The steering angle is shown in Figure 4 and $\mathrm{v}=30 \mathrm{~km} / \mathrm{h}$, the vehicle's roll angle is determined through the figure above.

Since the car moves at low velocity, the vehicle's roll angle is not large, so the active stabilizer bar hasn't been activated. With the above conditions, if the velocity increases to $60 \mathrm{~km} / \mathrm{h}$, the active stabilizer bar will be activated. At this time, the electric motor (hydraulic) will produce the torque impacting to two parts of the bar, reducing the vehicle's roll angle. However, at $\mathrm{v}=60$ $\mathrm{km} / \mathrm{h}$, the stabilizer bar is only activated at mode 1 , so the angle $\varphi_{\mathrm{x}}$, when equipped active stabilizer bar, is only equivalents to the passive stabilizer bar (Figure 10).

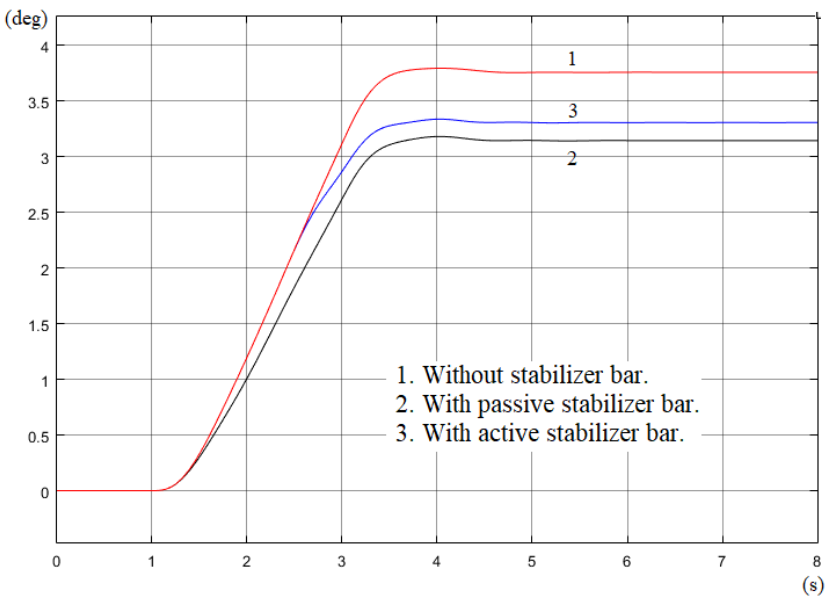

Figure 10: Roll angle at $\mathrm{v}=60 \mathrm{~km} / \mathrm{h}$

In case the vehicle moves at $\mathrm{v}=120 \mathrm{~km} / \mathrm{h}$ and the center height is also increased, the value of $h_{1}=0.6 \mathrm{~m}$. The active stabilizer bar is activated at mode 2 , the vehicle's roll angle $\varphi_{\mathrm{x}}$ is significantly reduced (Figure 11).

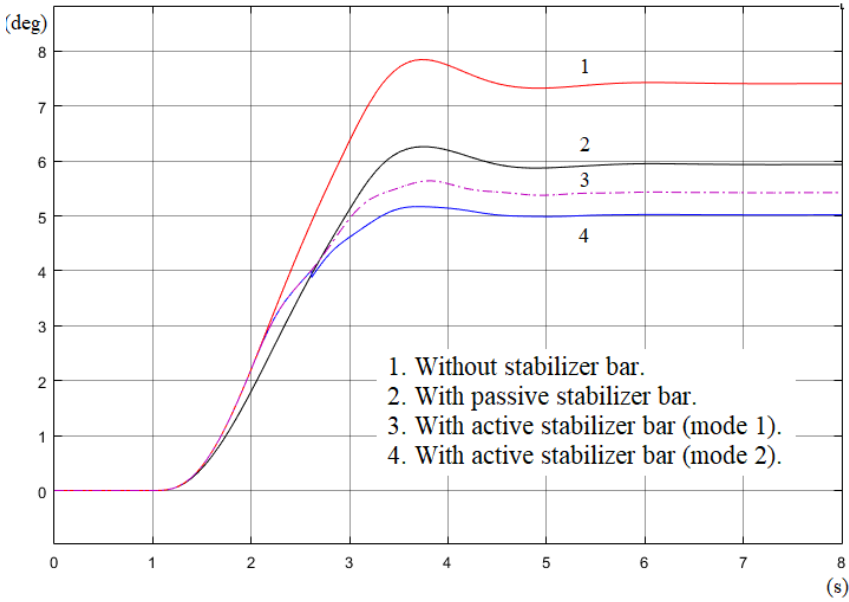

Figure 11: Roll angle at $\mathrm{v}=120 \mathrm{~km} / \mathrm{h}$

With the above moving conditions, if the vehicle isn't equipped with a stabilizer bar, the vehicle can rollover. When the passive stabilizer bar is equipped, the vehicle's roll angle can be reduced, but because the stiffness of the bar is constant, it hasn't fully utilized the effect of the bar. In case, it can be seen that the active stabilizer bar has superiority over both cases.

\section{Conclusion}

The rollover phenomenon usually occurs when the roll angle is too large. This value depends on the velocity, center height, and steering angle of the vehicle. When the vehicle steers with high velocity, it is very easy to appear in this situation, the vehicle needs to be equipped stabilizer bar to limit this phenomenon. Research has shown that the vehicle's roll angle is significantly reduced when equipped stabilizer bar and shown the outstanding advantages of the active stabilizer bar compared to the passive stabilizer bar.

In the case of the vehicle's roll angle is small, the active stabilizer bar doesn't activate, so the smoothness of the vehicle will not be affected. When the value $\varphi_{\mathrm{x}}$ reaches the required threshold, the bar will activate and act as active stabilizer bar. In the case of roll angle is too big, the bar will switch to the second mode to significantly reduce the vehicle's roll angle, thereby making the vehicle more stable.

The study only focused on establishing the dynamic vehicle model equipped stabilizer bar and conducting simulation by Matlab - Simulink software. The parameters are referenced based on actual vehicles, the simulation was conducted under ideal conditions and not to mention the influence of external factors. Therefore, it is necessary to have empirical verification to make accurate statements.

\section{References}

[1] N. T. Anh, T. T. Tran, H. T. Binh, P. H. Nam, L. T. Dung, "Study on the Method of Calculating and Designing Stabilizer Bar on the Vehicle Using Solidworks Software", Viet Nam Mechanical Engineering Journal, 7(12), 9299, 2018.

[2] N. K. Trai, N. T. Hoan, H. H. Hai, P. H. Huong, N. V. Chuong, T. M. Hoang, Structure Vehicle, Bach Khoa Ha Noi Publishing, 2010.

[3] P. Bravin, T. Kshitijit, P. Amit, K. Ganesh, "Design, Analysis and Optimization of the Anti-roll Bar", Journal of Engineering Research and Applications, 4(9), 137-140, 2014.

[4] A. N. Khartode, M. U. Gaikwad, "Design and Analysis of Anti-roll Bar for Automotive Application", International Journal on Recent and Innovation Trends in Computing and Communication, 4(6), 340-345, 2016.

[5] P. H. Dong, "Establish on the Lateral Roll Model of the FAW 29 Seats, Produce and Assemble in Viet Nam", Master Thesis, Ha Noi University of Science and Technology, 2015.

[6] J. N. Husen, A. M. Naniwadekar, "Analysis of Anti-roll Bar of a Passenger Car for its Nonlinear Behavior with Help of CAE", International Journal of Science Technology \& Engineering, 2(1), 133-136, 2015.

[7] V. V. Huong, N. T. Dung, D. N. Khanh, D. H. Phuc, Dynamics Vehicle, Viet Nam Educational Publishing, 2014.

[8] G. A. Hassaan, N. A. Mohdsamin, "Vehicle Dynamics Response to Road Hump Using a 10 Degrees of Freedom Full-car Model”, International Journal of Computer Techniques, 2(1), 56-62, 2015.

[9] V. Muniandy, P. Mohdsamin, H. Jamaluddin, R. A. Rahman, S. A. Abubakar, "Double anti-roll bar hardware-in-loop experiment for the active anti-roll control system", Journal of Vibroengineering, 19(4), 2886-2909, 2017

[10] N. T. Anh, "Research the Dynamic Vehicle Model Equipped Active Stabilizer Bar", Master Thesis, Ha Noi University of Science and Technology, 2019. 